\title{
MOTS DITS SOIENT LES MAUX : PAROLES POST-ABITUM DANS LE RECUEIL « DESAPARIENCIA NO ENGaÑA » DE NÉSTOR PONCE
}

\author{
Orianne GUY \\ ERIMIT-Université de Rennes 2
}

\begin{abstract}
The purpose of this article is to introduce an analysis of Néstor Ponce poems compiled in the book «Desapariencia no engaña » (2010). Forced to flee the country to escape the dictatorship (1976-1983), the Argentinian writer went into exile in France in 1979. With the restoration of democracy, the author did not return to his home country but continued to live in France. More than twenty years after the dictatorship, Néstor Ponce defies space and time to return to that tragic moment in history and takes his pen to remember. Never to forget « los desaparecidos » never to forget the places of torture, never to forget the horror, death. The author goes beyond a memorial work: he gives voice, in this versified present, to prisoners of the past so that they are recognized as 'victims-people' and not as 'desaparecidos-anonymous'. Néstor Ponce willfully ignores the political discourse and creates the neologism « desapariencia ${ }^{1}$ which is the association of two words: desaparición (disappearance) and apariencia (appearance). The writer uses words and poetry to respond to the oblivion speech imposed by the dictators.
\end{abstract}

Keywords : poetry; Argentina; dictatorship; missing people; memory

Mots-clés : poésie ; Argentine ; dictature ; disparus ; mémoire

\section{Introduction}

Vingt-sept ans après la chute du pouvoir dictatorial en Argentine (1976-1983), l'auteur Néstor Ponce défie l'espace et le temps pour revenir sur ce moment tragique de l'Histoire et prend sa plume pour ne pas oublier ${ }^{2}$. Ne pas oublier «los desaparecidos $»^{3}$, ne pas oublier les lieux de torture, ne pas oublier l'horreur, la mort. Le poète va au-delà du travail de mémoire : il offre la parole dans ce présent versifié aux prisonniers du passé afin qu'ils soient reconnus comme 'victimesindividus' et non comme 'desaparecidos-anonymes'. Rappelons ici le discours du général Videla qui niait la réalité et répondait que les disparus étaient «inconnus puisque disparus... Sans identité, ni morts ni vifs, puisque disparus... ».

Pour échapper au régime dictatorial, Néstor Ponce a été contraint de fuir son pays et s'est exilé en France en 1979. Au retour de la démocratie, l'auteur ne repart pas dans son pays d'origine mais continue sa vie dans l'Hexagone. Il a connu le manque et la perte des repères et sait à quel point la récupération de la mémoire est primordiale pour la reconstruction identitaire de la nation : la culture, ramifiée ou étouffée, doit se réapproprier l'Histoire et réciproquement. Pour ce faire, Néstor Ponce a décidé, non pas d'écrire l'expérience de l'exil, mais celle du silence.

\footnotetext{
${ }^{1}$ Le recueil a été traduit en français sous le titre de «Désapparences » par Monique Roumette.

${ }^{2}$ Pascal Gabellone (2011) expose une réflexion intéressante au sujet du temps du poète : «Il s'avère nécessaire, pour Reverdy, de révéler le temps propre du poète dans son rapport à l'événement et au politique. Son temps n'est pas celui de la simultanéité (...), mais bien celui du décalage, de l'attente subtile, de l'antériorité ou de la postériorité. Ce jeu avec le temps de l'événement n'implique en aucune manière une absence ni une indifférence envers celui-ci. », Poésie et politique au XX ${ }^{\text {ème }}$ siècle, dirigé par Henri Behart et Pierre Taminiaux, Paris, Hermann, p. 15.

${ }^{3}$ Traduction : les disparus.

Echo des études romanes XII/1-2, 2016 
Peinture et poésie sont des lieux de silence $^{4}$ : s'alternent ainsi, dans l'édition bilingue $^{5}$, des créations de l'artiste chilien Guillermo Nuñez ${ }^{6}$ et les poèmes de l'auteur argentin. Cette insertion d'œuvres picturales donne une dimension universelle à la représentation de la souffrance subie au $\mathrm{XX}^{\mathrm{e}}$ siècle.

C'est un projet audacieux que celui de créer une voix qui n'a pas pu trouver sa place dans le temps; des voix volées que l'auteur cherche à enraciner dans ses poèmes. Il est question en l'occurrence de fiction poétique car Néstor Ponce prend la voix de ces disparus ${ }^{7}$. De ce fait, l'écrivain recrée une mémoire qui a été rayée de l'Histoire argentine car les dictateurs voulaient effacer toute «trace » de subversion. Cet acte d'écriture est en soi une réponse qui résiste au discours autoritaire de l'époque. Pour contrer la violence verbale et physique, Néstor Ponce a choisi le Verbe poétique mêlé aux images suggestives de corps meurtris. Ainsi, l'auteur revalorise les mots qui ont été maltraités dans les discours des dictateurs. Cette idée nous rappelle le poème de Juan Gelman intitulé Regresos :

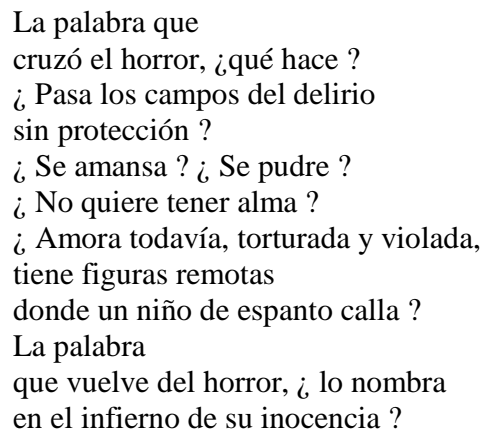

Néstor Ponce démontre au travers du titre «Desapariencia no engaña » que les Argentins ne se sont pas laissé leurrer et que la poésie permet la liberté de la parole, mais aussi la parole de la liberté. L'écrivain se joue ainsi du discours politique et crée le néologisme «desapariencia » qui est la fusion des deux termes initiaux : desaparición (disparition) et apariencia (apparence). S'entend sous ce jeu de mots : les disparitions ne sont en fait que des apparences. Trente-sept poèmes composent ce recueil. Cette écriture, qui fait fi de l'espace et du temps, invite le lecteur à réfléchir sur l'intemporalité du discours poétique ainsi que sur son rôle de « lieu de résistance ».

\footnotetext{
${ }^{4}$ Selon Laurent Fels (2009), « [...], la poésie trace le seuil entre le silence, la solitude et l'autre limite du mot. (...). Le poème s'écrit désormais dans le langage d'un cœur éclaté en silences. », Regards sur la poésie du XXème siècle, Namur, les Éditions namuroises, p. 7.

${ }^{5}$ Néstor Ponce (2013), Desapariencia no engaña / Désapparences, Brest, Le hauts Fonds.

${ }^{6}$ Guillermo Nuñez a reçu le prix national des Arts chiliens en 2007 et son œuvre exprime « les violences que l'homme peut infliger à l'homme ». Nous vous renvoyons à la présentation de l'artiste faite par Monique Blaquière Roumette dans sa note de lecture concernant l'ouvrage étudié.

${ }^{7}$ Voir l'entretien filmé de l'auteur à ce sujet, http://www.youtube.com/watch?v=L9ZTOuWwhBU, consulté le 17/02/14.
} 


\section{Réponse au discours autoritaire : l'écrit poétique}

Dès le premier texte, nous découvrons que les poèmes sont une réponse à l'autorité : ils agissent comme lieux d'insoumission ${ }^{8}$. Radio encendida nous propose une sorte de dialogue interstrophique ${ }^{9}$ : les quatre strophes 'principales', d'un point de vue quantitatif, sont séparées par ce que le prisonnier entend à la radio. Nous lisons entre parenthèses les paroles du tango «Mi Buenos Aires querido », et en toute fin de poésie, « (la radio decía : «Comunicado de la Junta Militar») ». La musique sert ici à «étouffer» les cris des prisonniers torturés. La violence prend place dans les vers au travers des champs lexicaux («lo partieron por el medio »; «le dieron electricidad »; « lo bombardearon ») et des barres obliques qui figurent la rupture (« lleno de dientes en la cabeza / pobre bicho loco »; « la reventaron / se lo juro señor / »). Mais la sentence est donnée avec ce coup de grâce final : « Militar », étant le dernier mot du poème, s'impose en tant qu'autorité qui vient arrêter toute forme poétique, en l'occurrence le chant de Gardel à la radio ainsi que le texte de Néstor Ponce. À l'inverse, la fin du poème empêche le lecteur de découvrir ce que la Junte va énoncer à la radio : de cette manière, le poète clôt le discours qui se présente comme étant autoritaire. Outre cela, l'utilisation de la barre oblique, servant ici d'idéogramme, n'est pas sans rappeler l'écriture de Juan Gelman, auteur argentin lui aussi ayant connu l'exil ainsi que la perte de ses proches. Ce dernier a dédié nombre de ses poèmes à l'absence et nous pensons ici au recueil Interrupciones II, dans lequel cette stratégie typographique est révélatrice visuellement de cette cassure dans une vie ${ }^{10}$. Par-delà la stratégie discursive, Néstor Ponce fait appel aux références culturelles de l'Argentine et donne leur place à Carlos Gardel, Juan Gelman ou encore Osvaldo Soriano, écrivain argentin exilé lui aussi, qui avait lui-même intitulé un de ses romans No habrá más pena ni olvido, parole du tango cité dans le poème. Le pacte de lecture est ainsi donné : l'intertextualité permet de renforcer le discours poétique en affirmant son appartenance à une culture préexistante à la dictature et ayant survécu à cette dernière.

Le douzième poème, intitulé Interrogatorio, fait écho, quant à lui, aux interrogatoires faits aux Argentins soupçonnés d'être «subversivos ». La série de questions impose la répétition des points d'interrogation à l'envers qui, de ce fait, deviennent la métonymie anaphorique de la question, de l'incertitude, du silence. Le poème commence avec une question pouvant être posée par les paramilitaires ( « ¿Cuál es tu nombre o tu hambre de guerra ? ») puis il s'oriente, dès le deuxième vers, en direction de la question poétique, qui peut paraitre surréaliste dans ce contexte de violence : « $¿$ De qué color los ruiseñores los versos del viejo Martín ? ». Cette question est en réalité une arme de défense : le prisonnier répond par une

\footnotetext{
${ }^{8}$ Dans «La poésie, c'est autre chose », mille et une définitions de la poésie, nous nous arrêtons sur ces phrases : "Résistance, révolte, insurrection spirituelle. La poésie est d'abord cela : acte d'insoumission. », Paris, Arfuyen, p. 17.

${ }^{9}$ Cf. l'étude de Marián Semilla Durán concernant la structure des poèmes gelmaniens reproduisant « la tension dialectique entre la vie et la mort », «De pájaros y hojas : una poética de la resurrección » in La memoria de la dictadura, dirigé par Fernando MoReno (2006), Paris, Ellipses, p. 295-303.

${ }^{10}$ Voir à ce sujet les études réunies dans le livre La memoria de la dictadura, dirigé par Fernando MoReno (2006), Paris, Ellipses.
} 
question qui sert de bouclier poétique et renvoie à l'histoire littéraire de l'Argentine. En effet, le prénom cité pourrait renvoyer au Martín Fierro, personnage gauchesque de l'œuvre poétique de José Hernández, considéré comme ouvrage majeur de la littérature argentine ${ }^{11}$. Puis, de nouveau se lisent les interrogations concernant la réalité de la terreur (« $i$ Direcciones nombres citas ? « $i$ Por qué se vacían las calles ?»). Cette saturation de questions impose une lecture haletante, mais aussi angoissante de par l'absence de réponse. Or, le texte est lui-même réponse au discours autoritaire et transmet au lecteur cette expérience du silence. Nous retrouvons une allusion à ce poète fictif surnommé Martín dans le poème intitulé Poemas del viejo Martín où les mots sont personnifiés, où ce sont les vers qui subissent la terreur du Processus ${ }^{12}$. Dans ce texte, l'auteur compare deux dictatures : celle d'Hitler qui brûlait les livres, pour faire disparaître la culture, la mémoire collective, et celle imposée en Argentine (« los quemaban en Berlín », « los ejecutaban en La Plata »). Au travers de ce poème, Néstor Ponce rend hommage aux écrivains victimes de la répression ${ }^{13}$.

Dans Fiesta en el campo, les strophes s'articulent sur deux espaces décalés ce qui nous fait penser de nouveau à un dialogue entre le prisonnier qui s'interroge et l'horreur de l'armée meurtrière. Les vers attribués à la violence sont plus nombreux que ceux dédiés au regret, à l'amertume. La répétition des termes «pero » et « tanto », les homophonies ainsi que l'allitération du son [o] sont significatives du désarroi du prisonnier face à l'horreur. Je cite :

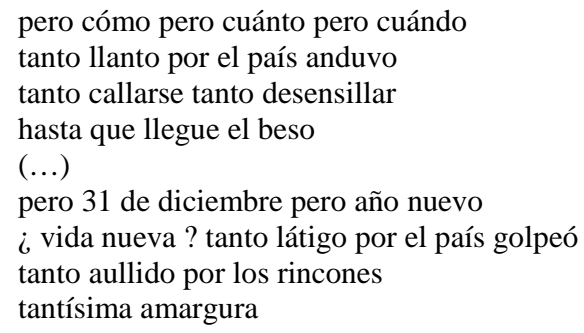

Nous notons dans cet extrait l'absence de points d'interrogation dans la première strophe ce qui peut signifier l'infini, l'atemporalité des questions qui restent sans réponses ; tandis que leur présence dans la seconde strophe sert à mettre en relief l'espoir misé sur cette nouvelle année qui commence, 1977 d'après l'indication écrite après le titre. Nous reviendrons d'ailleurs, plus loin dans l'étude, sur ces détails significatifs que sont les lieux et dates inscrits au début de chaque poème. Outre le caractère destructeur de la terreur, Néstor Ponce s'arrête dans la dernière strophe sur cette question fondamentale qu'est la civilisation versus la barbarie. Ladite civilisation des dictateurs, le « processus de réorganisation nationale », est

\footnotetext{
${ }^{11}$ HeRnÁndeZ José (1989) ( $1^{\circ}$ éd. 1872), Martín Fierro, Madrid, Alianza Editorial.

${ }^{12}$ Le terme «Proceso » est un euphémisme inventé par les intellectuels argentins qui ne pouvaient pas parler ouvertement de « dictature » entre 1976 et 1983.

${ }^{13}$ Le poème Ronda est dédié à Rodolfo Walsh, écrivain et journaliste enlevé par les militaires et dont le corps n'a jamais été retrouvé.
} 
comparable à la soi-disant barbarie des Indiens ${ }^{14}$. L'horreur sanguinaire a perduré au travers des cycles de l'Histoire. Rappelons que cette question concerne aussi le pouvoir dominant du $\mathrm{XVI}^{\mathrm{e}}$ siècle en Amérique, c'est-à-dire les colons espagnols, qui ont décimé nombre de peuples autochtones, qu'ils considéraient alors comme barbares.

Enfin, en réponse au discours autoritaire, nous relevons le poème intitulé Carnaval. En effet, ce thème renvoie à la fantaisie voire au ridicule : Mikhaïl Bakhtine y déchiffrait le renversement des hiérarchies dans l'œuvre de Rabelais ${ }^{15}$. C'est le monde à l'envers, la subversion de la réalité. En plus de ce terme, Néstor Ponce glisse dans les vers les substantifs «máscaras », «teatro griego » et «murga tambor y redoble ». Ce champ lexical de la représentation rend les agents militaires grotesques : la poésie fait un affront à la dictature en la ridiculisant. Ici, les mots font un pied de nez à l'autorité et questionnent encore le lecteur concernant la frontière entre réalité meurtrière et imaginaire insensé : où est le sens de cette folie ?

\section{Une trace de l'oubli}

Outre cette réponse, visible et lisible, faite au discours autoritaire, la poésie se veut résistante à l'oubli. L'écriture est en soi une trace, une marque dans le temps et s'insère dans la mémoire, individuelle et/ou collective ${ }^{16}$. Marián Semilla Durán l'exprime en ces termes :

Poesía y memoria operan una doble alianza : a la vez archivos - cementerios - simbólicos y espacios de resurrección, la letra y la imagen constituyen un dispositivo complejo que devuelve a los muertos sus atributos humanos, que personaliza las listas de cadáveres anónimos y sustituye la lógica intimista de los afectos. ${ }^{17}$

Si les dictateurs ont tout fait pour faire disparaitre los « subversivos », bannir de leur temps ceux qui leur semblaient dérangeants pour la bonne conduite du pays, la poésie, pour sa part, les réintègre dans ce nouvel espace-temps rhétorique et mémoriel. Cela rappelle le désir de Jorge Semprún qui demandait «que les écrivains s'emparent de la mémoire de la mort, sinon elle n'existerait plus... ». Les hommes disparaissent mais les écrits restent.

Au-delà de l'acte d'écriture en lui-même, Néstor Ponce donne à ces voix l'occasion de s'exprimer sur la mémoire et l'oubli. Trois textes nous semblent révélateurs de cette question et deux d'entre eux ont des titres explicites : Memoria perra fiel et Yo también dicto cartas de memoria a mi mamá. Nous y ajoutons le poème Objetos personales dans lequel le prisonnier énumère les affaires qui pouvaient être prises par les militaires et conclut ainsi :

\footnotetext{
${ }^{14}$ Ceci renvoie à Walter Benjamin qui propose de reconsidérer l'Histoire depuis le point de vue de l'Autre.

${ }^{15}$ BAKHTINE Mikhaïl (1982), L'œuvre de François Rabelais et la culture populaire au Moyen-Âge et sous la Renaissance, Paris, Gallimard.

${ }^{16}$ Rappelons ici le rôle que la poésie joue pour la mémoire dans l'histoire littéraire : les vers étaient mémorisés et répétés inlassablement, au gré des transformations. L'écriture a donc renforcé cette caractéristique de la poésie en fixant la mémoire sur papier.

${ }^{17}$ Semilla DuRÁn Marián, Op.Cit., p. 296.
} 


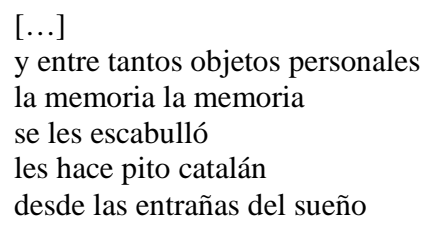

ni olvido ni perdón

La répétition du terme «memoria » s'inscrit dans le champ visuel du lecteur et donne aussi l'impression de vouloir se graver dans sa mémoire. Les militaires n'ont pas pu voler la mémoire, grâce aux témoignages, grâce à l'écriture mais aussi grâce aux mesures d'État ${ }^{18}$. S'ils ont tenté de la prendre et de forcer l'oubli, le prisonnier conclut, avec ce vers final, imposant, que pour sa part il n'oubliera pas l'Histoire et il ne pardonnera pas. La «memoria » doublée semble plus forte que «el olvido» nommé une seule fois.

Dans Memoria perra fiel, il est question de nostalgie : celle du temps passé et celle du temps désiré. Dans ce poème, le «calendrier s'effeuille », «les caresses mortes fendillent la date des retrouvailles » et les souvenirs sont $«$ en flammes $»^{19}$. Ce champ lexical, renvoyant à la saison automnale, symbolise la conscience de la défaite chez le prisonnier : le temps n'a plus sa place dans le camp de concentration où il est retenu. On devine toutefois à l'avant-dernier vers que, malgré ce sentiment de désespoir infini dans le temps, l'amour est toujours « encadré » par cette attente comme nous pouvons l'observer :

Te espero amor te espero

Para compartir los jugos de este tiempo

La mémoire est fidèle mais les souvenirs font souffrir le prisonnier, renforçant alors l'image de l'absence de l'autre.

Dans Yo también dicto cartas de memoria a mi mamá, le prisonnier nous annonce qu'il se dicte des cartes de mémoire : la trace n'est pas écrite mais elle s'inscrit dans la mémoire et permet de faire «barrage contre l'épouvante », de lutter contre l'oubli et le silence. De ce fait, les lettres non mises sur papier sont libres et se mélangent : les souvenirs heureux côtoient l'horreur quotidienne. Ces mots trouvent leur place dans le texte que nous avons sous les yeux. Le bonheur perdu ne se laisse plus dominer par les maux mais prend consistance parmi eux. Observons l'emploi des parenthèses pour «con dulce de leche »: ordinairement, cette typographie est principalement utilisée pour ajouter des données secondaires à un texte. Or, à la première vue du poème, ce détail saute aux yeux du lecteur : le 'doux' souvenir y trouve une place centrale. Dans la seconde strophe, il est question d'oubli forcé et de perte. Le prisonnier, face à l'oubli et aux tumultes des lettres, se perd dans la tristesse et finit ainsi : «los extraño mucho mamá los extraño ». Le sentiment

\footnotetext{
${ }^{18}$ Nous pensons ici aux procès ouverts suite à la décision du président Néstor Kirchner (décret datant de 2004). Cette reconnaissance de l'horreur a permis d'installer une tradition de la mémoire.

${ }^{19}$ Nous reprenons ici la traduction de Monique Roumette dans Désapparences.
} 
de manque, réitéré, essaie de récupérer la mère en l'entourant sur ce dernier vers tout comme le prisonnier aurait souhaité être enlacé par su «mamá ».

Nous avons vu jusqu'à présent le rôle de la poésie face au discours autoritaire et face à l'oubli : Néstor Ponce écrit en jouant avec la forme du poème, la typographie ou encore la répétition de mots-clés qui doivent marquer le lecteur et permettre aux paroles des prisonniers de bien trouver leur place dans ce recueil. Nous allons approfondir cette recherche en analysant de plus près tous ces détails qui font que la poésie est en soi un lieu de résistance.

\section{Lieu de résistance}

Nous y avons fait allusion précédemment : chaque poème est accompagné d'un chronotope. Cette stratégie poétique permet de fixer davantage ces prisonniers inconnus dans l'espace textuel : on ne connaît pas leurs noms mais on sait qu'ils ont été enfermés à La Escuelita, Bahía Blanca, à La Cueva, à la ESMA ou bien encore au Campo de Mayo. Les dates s'échelonnent de 1976 à 1980, si l'on exclut le dernier poème qui relève de l'autorité de Néstor Ponce et non d'un prisonnier imaginé. Ces repères spatio-temporels prennent place dans ce recueil, en haut à droite des pages, tel qu'on pourrait le faire quand on envoie une lettre. Ces données sont la preuve de l'existence de ces prisonniers anonymes ${ }^{20}$.

Par ailleurs, les poèmes sont écrits en vers libres, réitèrent les enjambements ou encore les hyperbates : ici le poète semble vouloir libérer ces mots de la norme comme s'ils pouvaient s'affranchir de ces camps de concentration. Conjointement à cette idée, nous découvrons justement à plusieurs reprises le champ lexical de l'oiseau, symbole de la liberté : « un pajarito », « La paloma volante », « ruiseñor », « la paloma de Picasso », « golondrinas », « los gorriones » ou encore « pájaro ». Face à ces animaux de paix qui « [éclatent] en plein vol », nous lisons « los lobos ${ }^{21}$, ceux qui sortent la nuit pour chasser sous la lune, ou encore « las víboras que reptan el país $»^{22}$.

Nous remarquons aussi l'absence quasi permanente de ponctuation, exception faite de la saturation des points d'interrogation que nous avons vue précédemment. Les majuscules sont utilisées par endroits, en général en début de strophe, ou pas du tout (nous pensons ici aux poèmes Estrellas en el Río de la Plata, Fin de semana en la costa ou encore Variaciones (recuerdos)). Ce choix implique une lecture active, déjà impulsée par le caractère poétique des textes. En effet, si les majuscules semblent être mises de manière aléatoire, nous ajoutons que les points finaux ne font

\footnotetext{
${ }^{20}$ Notons l'observation intéressante dans la note de lecture d'Eric Courthès : « Cette mention exacte du centre de détention ou du camp de concentration d'où sort la voix de la victime est maintenue jusqu'à l'avant-dernier poème : 'Corredor de la espera', quand brusquement l'auteur change et mentionne : 'cerca de tu casa, mañana por la mañana', laissant à entendre au lecteur que le totalitarisme peut frapper n'importe où et n'importe quand, et nous impliquer directement... » Et dans le dernier poème, «Gota de belleza », il mentionne tous les lieux par lesquels il est passé, de l'Argentine vers la France, inscrivant pour la première fois son moi, de manière élégante et discrète dans son ouvre... »; Dans son texte, Jean-Marie Lassus porte sa réflexion quant aux noms trompeurs des lieux cités.

${ }^{21} \mathrm{Cf}$. les poèmes Fugas, p. 30 et Terremotos en los ojos, p. 82.

${ }^{22}$ Cf. le poème Tránsito, p. 66.
} 
pas partie de l'écriture de Néstor Ponce dans ce recueil. Nous en repérons deux dans le poème Fugas, à la cinquième strophe :

«La tarde huele a pavimento en verano y de pronto el grito.

Lo ven caer, volarse, hacerse. Ahí viene »

Le point signifie la fin, la mort. Or, ces poésies sont un cri «emparolé », qui ne veulent cesser d'exister donc les points finaux n'ont pas leur place dans les textes. Ce choix esthétique engendre aussi un essoufflement dans la lecture, une sorte de discours poétique sans fin qui résiste au temps.

D'un point de vue typographique, nous avons vu que le poète avait régulièrement recours à la barre oblique qui viendrait remplacer cette idée de coupure dans la phrase, dans l'idée, dans le bruit, tout en préservant l'unité du poème. Outre ce procédé, Néstor Ponce donne sens à certaines de ses poésies en rendant leur présentation visuelle harmonieuse avec leur contenu. Pour exemple, nous pouvons nous arrêter sur le poème $25^{\circ}$ aniversario de casados en el campo qui est le plus révélateur de cette stratégie au biais du calligramme. En effet, le prisonnier semble chuter dans ce précipice, cette brèche que lui impose la dictature : à la lecture, les yeux du lecteur chutent eux aussi au regard des mots car ceux-ci sont écrits « en escaliers » :

Sobre este hueco

desde


Sobre este agujero

esta

cornisa

Les mots semblent vivre l'expérience du prisonnier et communiquent cette chute au lecteur.

Enfin, une autre technique qui sous-entend la résistance dans la poésie est la saturation : nous trouvons en effet une redondance régulière de lexiques renvoyant à la belle mort, l'absence de l'autre, le cri, la souffrance (psychique et physique) ou encore le temps. Cette réitération lexicale ressasse les pensées, comme pour combler un vide. Toutefois, le poète a recours à des images qui embellissent la mort de ces disparus. Nous pensons ici à l'eau et à l'argent : el Río de la Plata. Ce fleuve qui porte un nom si joli est pourtant un des hauts lieux de crimes de cette dictature : les corps engourdis par la drogue y étaient jetés depuis les avions ; d'ailleurs un des poèmes, Traslado, a pour originalité d'avoir été « localisé » dans un de ces avions. Avec Néstor Ponce, les corps balancés deviennent des étoiles, des cheveux d'argent, des colombes d'argent, les cris, eux, sont des carillons de lumière.

La prière et la litanie renforcent cette saturation : la répétition de mêmes vers ou de séries de mots oblige le lecteur à assimiler le discours poétique proposé. En sont 
les exemples parfaits les poèmes Rezo una pequeña plegaria et Cumpleaños en el campo dont voici un extrait explicite :

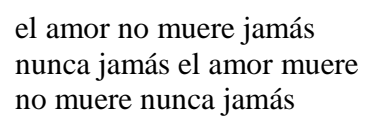

Dans ce poème, le prisonnier semble vouloir se marteler ces mots pour les préserver de l'oubli et rester convaincu de cette vérité.

Enfin, nous retrouvons l'emploi de l'intertextualité dans les poèmes Cumpleaños en el campo et $25^{\circ}$ aniversario de casados en el campo qui semblent se répondre, se compléter, et l'apparition de repères culturels dans certains poèmes renforce le discours de celui-ci qui résiste à la dictature comme nous avons pu le voir précédemment.

\section{Conclusion}

Au travers de ces poèmes, Néstor Ponce a voulu politiser la littérature et non pas écrire des pamphlets. Les cagoules, la torture, les disparitions n'ont pas pu empêcher cette quête de justice, de réajustement de l'Histoire qui avait été déviée. Néstor Ponce nous le prouve dans ce recueil de poésies exceptionnel : de par son audace, de par cette volonté de restituer une place à ces voix, de par la polyphonie, de par l'hybridation énonciative, de par cette saturation omniprésente et cette liberté de parole qui veut s'ancrer dans la mémoire collective. Parallèlement, Néstor Ponce fait usage de la mémoire exemplaire en dénonçant un passé de l'horreur : celui du $\mathrm{XVI}^{\mathrm{e}}$ où les Espagnols ont décimé des peuples indigènes mais aussi celui plus récent $\mathrm{du} \mathrm{XX}^{\mathrm{e}}$ siècle, le siècle des dictatures.

Dans son écriture, les gouttes de sang deviennent des « gouttes de temps », des « goutte[s] emplie[s] de lumière » et enfin des « goutte[s] de beauté ». Face à cette beauté de la poésie, l'horreur est écrite, est peinte. Elle a aussi été mise en musique et lue à voix haute à de multiples reprises ${ }^{23}$. De ce fait, la résistance de l'art poétique va au-delà de son support écrit : elle invite les autres arts et nous-mêmes lecteurs à venir résister face au silence, face à l'oubli.

\section{BIBLIOGRAPHIE}

BAKHTINE Mikhaïl (1982), L'œuvre de François Rabelais et la culture populaire au Moyen-Âge et sous la Renaissance, Paris, Gallimard.

Behart Henri ; TAminiauX Pierre (éd.) (2011), Poésie et politique au XX siècle, Paris, Hermann.

BLAQUIÈRE-ROUMETTE Monique, http://www.recherchesinternationales.fr/RI95/RI95NLPonce.pdf, consulté le 10/03/2014.

\footnotetext{
${ }^{23}$ Le poème El otro caballo del general a été mis en musique par Higinio Mena ; Fugas et Gota de la belleza par Yves Krier.
} 
BLAQUiÈRE-RoumetTE Monique, Le général Videla les avait fait disparaître, Nestor Ponce redonne vie aux milliers d'opposants victimes de la dictature, http://www.humanite.fr/tribunes/le-general-videla-les-avait-fait-disparaitrenesto-541738, consulté le 10/03/2014.

ChOUCIÑo FERnANDEZ Ana (2016), Voces para la memoria sin límite : la obra poética de Néstor Ponce, Bulletin of Hispanic Studies, Volume 93, p. 667-681.

COURTHES Eric, « Désapparences » ne trompent pas, http://salonlitteraire.com/fr/societe/review/1841676-desapparences-ne-trompentpas?fb_action_ids=10151706050863836\&fb_action_types=og.likes\&fb_sourc e=aggregation\&fb_aggregation_id=288381481237582, consulté le 10/03/14.

FELS Laurent (2009), Regards sur la poésie du XX siècle, Namur, les Éditions namuroises.

GAultiER Maud (2011), Exploraciones futuristas del pasado en Azote de Néstor Ponce, Amerika [En ligne], 4 | 2011, mis en ligne le 30 mai 2011, consulté le 10 mars 2014. URL : http://amerika.revues.org/2110; DOI : 10.4000/amerika.2110

GUILLARD Amandine (2013), Resistencia y poesía en las cárceles argentinas (19761983), Amerika [En ligne], 8 | 2013, mis en ligne le 21 juin 2013, consulté le 10 mars 2014. URL : http://amerika.revues.org/3904; DOI : 10.4000/amerika.3904

HERNÁNDEZ José (1989) (1º́d. 1872), Martín Fierro, Madrid, Alianza Editorial.

LE CORRE Hervé (2013), Désapparences / Desapariencia no engaña, Les Langues Néo-Latines, $\mathrm{n}^{\circ}$ 366, septembre 2013, p. 131-133.

LIMA Rogério (2013), O livro do poeta : memórias e experiências pessoais de um período desleal, Amerika [En ligne], 9 |2013, mis en ligne le 20 décembre 2013, consulté le 10 mars 2014. URL : http://amerika.revues.org/4171; DOI : 10.4000/amerika.4171

MORENO Fernando (éd.) (2006), La memoria de la dictadura, Paris, Ellipses.

PFISTER Gérard (éd.) (2008), «La poésie, c'est autre chose », mille et une définitions de la poésie, Paris, Arfuyen.

PONCE Néstor (2013) (1 éd. 2010), Desapariencia no engaña / Désapparences, Brest, Le hauts Fonds, trad. Monique Roumette.

PONCE Néstor (2011), Memorias y cicatrices. Estudios sobre literatura latinoamericana contemporánea, Xalapa, México, Universidad Veracruzana.

SALERno María Paula, Néstor Ponce / Culinarias / Económicas / Médicas / Deportivas, http://www.abacq.org/cuaderno/index.php?Nestor-ponce-culinariaseconomicas-medicas-deportivas, consulté le 10/03/14.

WILDNER Tobias (2012), Recordar escribiendo : Gedächtnis und Erinnerung bei Néstor Ponce und Eduardo Belgrano Rawson, München, Meidenbauer. 